\title{
A novel geometric error identification methodology for the tilting head of five-axis machine tools based on double ball bar
}

\author{
Jinwei Fan, Changjun Wu, Zhongsheng Li \\ College of Mechanical Engineering \& Applied Electronics Technology, Beijing University of Technology, Beijing100124, China
}

\begin{abstract}
The paper proposes a novel geometric error identification methodology for the tilting head of five-axis machine tools using double ball bar(DBB).Firstly, based on the motion condition of the tilting head of five-axis machine tools, three measurement patterns in $\mathrm{Y}$ direction, $\mathrm{X}$ direction and $\mathrm{Z}$ direction are proposed respectively. Then, the relative displacement equations of two balls of DBB in three measurement patterns are established respectively on the basis of homogeneous transform matrix (HTM) and multi-body system (MBS) theory. Finally, the geometric error parameters of the tilting head are identified totally. The presented method is universal and provides a reference for the error identification for the tilting head of five-axis machine tools.
\end{abstract}

\section{Introduction}

With a rapid development of precision machining for the complex parts, five-axis machine tools (FAMTs) are preferred with the advantages of higher material removal rate, better the ability of positioning and orienting the tool with respect to the workpiece and lower production cost. However, the existing FAMTs cannot provide the same machining accuracy as their three-axis counterparts due to the additional rotary axes leading more geometric error sources, thus hindering the development and practical implementation of five-axis precision machining.

Over the past few decades, many researchers have investigated some efficient calibration approaches to identify the geometric error components of FAMTs based on double ball bar (DBB), which has been proved to be competent for rotary axes calibration. Zargarbashi et al. [1] presented a method to measure the position-dependent geometric errors of a trunnion-type A-axis using a DBB. Lee et al. [2] measured position-independent geometric errors of a rotary table axis(C-axis) and a trunnion axis (A-axis) of a five-axis machine tool using a DBB. Zhu et al. [3] proposed a new identification approach to recognize geometric errors of an A-tilting table by using a DBB. Zhang et al. [4] developed a novel measuring method to identify five geometric errors caused by the rotary table (C-axis) with a DBB. Lasemi et al. [5] provided a theoretical method to identify geometric errors of a rotary axis and $\mathrm{C}$ rotary axis utilizing a DBB. Chen et al. [6] proposed a comprehensive geometric error identification method for a tilt table using a DBB.

As can be observed in the above-mentioned researches, the geometric error identification methods for rotary axes of FAMTs are rarely aimed at the tilting head (B-axis). Therefore, an adequate geometric errors identification methodology for the tilting head (B-axis) of FAMTs should be developed.

\section{The measuring principle based on the DBB}

As shown in Figure 1, Every DBB measurement system has two sockets. The one clamped by the tool holder on the spindle is denoted as Socket 1 , and the other set on the worktable is denoted as Socket 2. Two ball of the DBB, which is denoted as Ball 1 and Ball 2 respectively, are installed on the ball bowls of the sockets. The measurement of the rotary axes is usually realized by detecting position changes of two balls of the DBB.

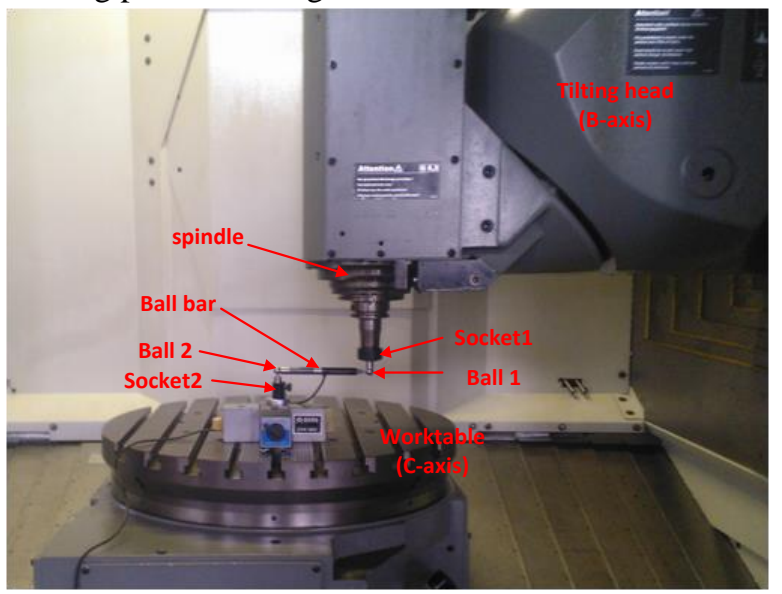

Fig.1 The measuring diagram for the tilting head based on DBB

According to Ref. [3], kinematic chain and homogeneous transform matrix (HTM) have been widely used as a general modelling method. In addition, Fan et al. [7] proposed a generalized kinematics modelling method, regarding a machine tool as multi-body system (MBS) 
including many rigid bodies. These theories can describe the motion relationship between adjacent bodies simply and conveniently (see Yang et al. [8] and Wu et al. [9]). Meanwhile, the position coordinates of two balls of the DBB can be transformed to the same coordinate system through kinematic chain respectively. Therefore, the mapping relationship between the theoretical model and actual measurement values can be established by calculating the distance between Ball 1 and Ball 2 .

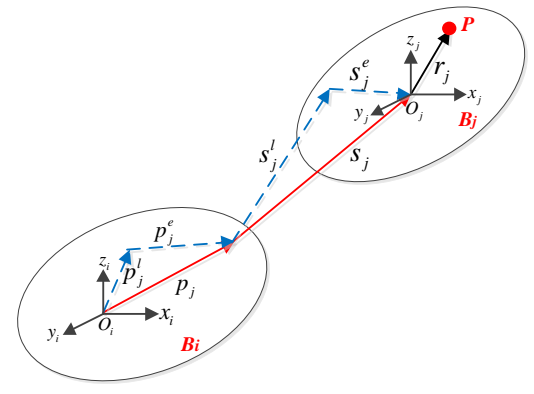

Fig.2 Schematic diagram of motion relation between the adjacent bodies

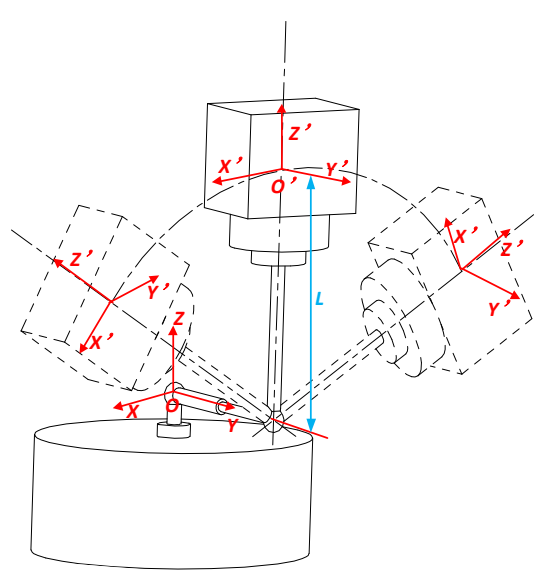

Fig. 3 The motion diagram of measurement pattern of $\mathrm{Y}$ direction for $\mathrm{B}$ axis

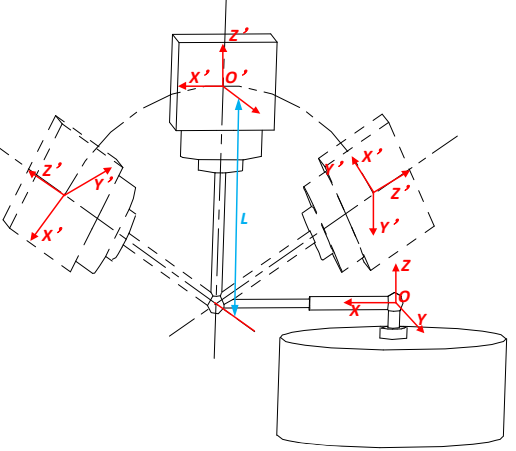

Fig. 4 The motion diagram of measurement pattern of $\mathrm{X}$ direction for $\mathrm{B}$ axis

As shown in Figure 2, the position array of $\mathrm{P}$ in the $B \mathrm{j}$ body coordinate system is represented as $\left\{r_{j}\right\}=\left\{\begin{array}{llll}r_{x} & r_{y} & r_{z} & 1\end{array}\right\}^{T}$, whereas the position array of $\mathrm{P}$ in the $\mathrm{Bi}$ body coordinate system is represented as $\left\{r_{j i}\right\}=\left\{\begin{array}{llll}x_{j i} & y_{j i} & z_{j i} & 1\end{array}\right\}^{T}$. Hence, $\left\{r_{j i}\right\}$ is described as,

$$
\left\{r_{j i}\right\}=\left\{p_{j}^{l}\right\}\left\{p_{j}^{e}\right\}\left\{s_{j}^{l}\right\}\left\{s_{j}^{e}\right\}\left\{r_{j}\right\}
$$

Where $\left\{p_{j}^{l}\right\},\left\{p_{j}^{e}\right\},\left\{s_{j}^{l}\right\},\left\{s_{j}^{e}\right\}$ describe the relative position transformation matrix, the relative position error transformation matrix, the relative motion transformation matrix, the relative motion error transformation matrix between the $B i$ and the adjacent lower body $B j$ respectively.

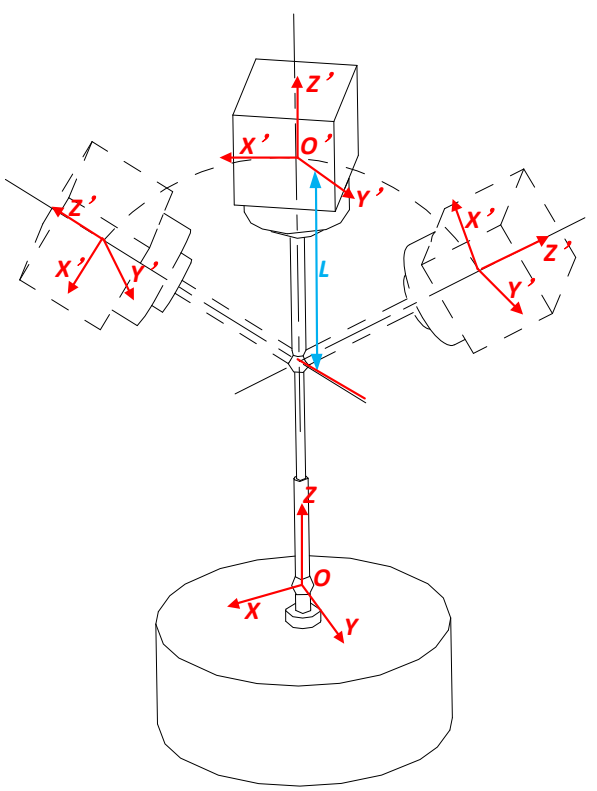

Fig. 5 The motion diagram of measurement pattern of $\mathrm{Z}$ direction for $\mathrm{B}$ axis

\section{Results and Discussion}

\subsection{The Identification Principle of Geometric Error Parameters}

Based on MBS theory, the tilting head (B-axis) has eight geometric errors, composed of six positiondependent geometric errors $\left(\delta_{x}(B), \delta_{y}(B), \delta_{z}(B), \varepsilon_{x}(B), \varepsilon_{y}(B)\right.$,

$\varepsilon_{z}(B)$ ), and two position-independent geometric errors $\left(\varepsilon_{x B}, \varepsilon_{B z}\right)$.

In the measuring process of the $\mathrm{DBB}, B$ axis is moving by multi-axes synchronous linkage to drive the spindle rotating around the centre point of Ball 1, whose position is required to keep unchanged. Figure 3-5 show three measurement patterns in $\mathrm{Y}$ direction, $\mathrm{X}$ direction and $\mathrm{Z}$ direction respectively. For the convenience of the research, the coordinate system of the centre point of Ball 2 is represented as $O-X Y Z$, whereas, the coordinate system of the gyration center of B axis is represented as $O^{\prime}-X^{\prime} Y^{\prime} Z^{\prime}$. Note that $O-X Y Z$ keeps stationary, and $O^{\prime}-X^{\prime} Y^{\prime} Z^{\prime}$ moves with the B axis. Fig.6 shows the position relation diagram of three measurement patterns. Due to the existence of geometric errors of B axis, the centre point of Ball 1 deviates from the deal position, denoted as $\mathrm{A}, \mathrm{C}$, and $\mathrm{D}$ respectively, to the actual position, denoted as A', C', and $\mathrm{D}^{\prime}$ respectively. Moreover, in $O^{\prime}-X^{\prime} Y^{\prime} Z^{\prime}$, the coordinate of $\mathrm{A}, \mathrm{C}$, and $\mathrm{D}$ is represented as, $\{A\}_{o}=\left[\begin{array}{llll}0 & 0 & -L & 1\end{array}\right]^{T},\{C\}_{o}=\left[\begin{array}{llll}0 & 0 & -L & 1\end{array}\right]^{T}$, 
$\{D\}_{o}=\left[\begin{array}{llll}0 & 0 & -L & 1\end{array}\right]^{T}$, where $L$ indicates the tool length between the gyration center of $\mathrm{B}$ axis and the centre point of Ball 1.
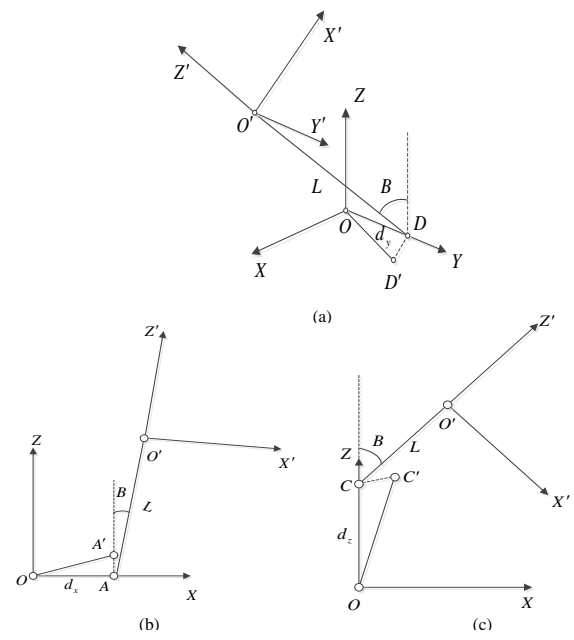

(a) Y-direction (b) X-direction (c) Z-direction

Fig. 6 The position relation diagram of three measurement patterns for B-axis.

\subsection{Measurement Pattern in Y Direction}

As shown in Figure 2 and 6(a), by utilizing (1), D' is described to $O-X Y Z$, then equation (2) is obtained.

$$
\begin{aligned}
\left\{D^{\prime}\right\}_{o} & =\left\{p_{j}^{l}\right\}\left\{p_{j}^{e}\right\}\left\{s_{j}^{l}\right\}\left\{s_{j}^{e}\right\}\{D\}_{o} \\
& =\left[\begin{array}{cccc}
1 & -\varepsilon_{x B} & 0 & 0 \\
\varepsilon_{x B} & 1 & -\varepsilon_{B z} & 0 \\
0 & \varepsilon_{B z} & 1 & 0 \\
0 & 0 & 0 & 1
\end{array}\right]\left[\begin{array}{cccc}
\cos B_{j} & 0 & \sin B_{j} & L \sin B_{j} \\
0 & 1 & 0 & d_{y} \\
-\sin B_{j} & 0 & \cos B_{j} & L \cos B_{j} \\
0 & 0 & 0 & 1
\end{array}\right] \\
& {\left[\begin{array}{cccc}
1 & -\varepsilon_{z}\left(B_{j}\right) & \varepsilon_{y}\left(B_{j}\right) & \delta_{x}\left(B_{j}\right) \\
\varepsilon_{z}\left(B_{j}\right) & 1 & -\varepsilon_{x}\left(B_{j}\right) & \delta_{y}\left(B_{j}\right) \\
-\varepsilon_{y}\left(B_{j}\right) & \varepsilon_{x}\left(B_{j}\right) & 1 & \delta_{z}\left(B_{j}\right) \\
0 & 0 & 0 & 1
\end{array}\right]\left[\begin{array}{c}
0 \\
0 \\
-L \\
1
\end{array}\right] }
\end{aligned}
$$

Where $B j$ indicates the rotating angle of $\mathrm{B}$ axis when the spindle moves from the initial position to the $j$-th position. $d_{y}$ indicates the original length of the ball bar in the measurement pattern of Y direction.

By simplifying Eq. (2) and ignoring the higher order term, the distance between $\mathrm{D}^{\prime}$ and $\mathrm{O}$ in coordinate system $O-X Y Z$ is able to be represented as, $L_{D^{\prime} O}=\sqrt{-2 L \sin B_{j} \varepsilon_{x B}+2 L d_{y} \cos B_{j} \varepsilon_{B z}+2 L d_{y} \varepsilon_{x}\left(B_{j}\right)+2 d_{y} \delta_{y}\left(B_{j}\right)+d_{y}^{2}}$,

Since $L_{D^{\prime} O}$ is equal to the actual length of the ball bar in the $\mathrm{j}$-th position, the following Equation can be obtained.

$$
\begin{aligned}
& \sqrt{-2 L \sin B_{j} \varepsilon_{x B}+2 L d_{y} \cos B_{j} \varepsilon_{B z}+2 L d_{y} \varepsilon_{x}\left(B_{j}\right)+2 d_{y} \delta_{y}\left(B_{j}\right)+d_{y}^{2}} \\
& =d_{y}+\Delta d_{y}
\end{aligned}
$$

Where $\Delta d_{y}$ indicates the length variation of the ball bar from the initial position to the $\mathrm{j}$-th position in the measurement pattern of $\mathrm{Y}$ direction.

Then, Equation (4) is obtained by simplifying (3) and ignoring the higher order term.

$$
L\left(\varepsilon_{B z} \cos B_{j}-\varepsilon_{x B} \sin B_{j}+\varepsilon_{x}\left(B_{j}\right)\right)+\delta_{y}\left(B_{j}\right)=\Delta d_{y}
$$

Assuming that $Q_{j}=\varepsilon_{B z} \cos B_{j}-\varepsilon_{x B} \sin B_{j}+\varepsilon_{x}\left(B_{j}\right)$,then equation (5) is obtained as follows,

$$
L Q_{j}+\delta_{y}\left(B_{j}\right)=\Delta d_{y}
$$

With two different values of $\mathrm{L}$, the following equation is obtained.

$$
\left\{\begin{array}{l}
L_{1} Q_{j}+\delta_{y}\left(B_{j}\right)=\Delta d_{y 1} \\
L_{2} Q_{j}+\delta_{y}\left(B_{j}\right)=\Delta d_{y 2}
\end{array}\right.
$$

According to Eq. (6), $\delta_{y}\left(B_{j}\right)$ and $Q_{j}$ are represented respectively as follows,

$$
\delta_{y}\left(B_{j}\right)=\frac{L_{2} \Delta d_{y 1}-L_{1} \Delta d_{y 2}}{L_{2}-L_{1}}, Q_{j}=\frac{\Delta d_{y 2}-\Delta d_{y 1}}{L_{2}-L_{1}}
$$

When $B_{j}=0^{\circ}$, i.e. $\mathrm{B}$ axis is at the initial position, $\varepsilon_{x}\left(B_{j}\right)=0$.Hence, $\varepsilon_{B z}=Q_{0}$.When $B_{j}=90^{\circ}$ or $270^{\circ}$, equation (8) is obtained.

$$
\left\{\begin{array}{l}
\left.Q_{j}\right|_{B_{j}=90^{\circ}}=-\varepsilon_{x B}+\left.\varepsilon_{x}\left(B_{j}\right)\right|_{B_{j}=90^{\circ}} \\
\left.Q_{j}\right|_{B_{j}=270^{\circ}}=\varepsilon_{x B}+\left.\varepsilon_{x}\left(B_{j}\right)\right|_{B_{j}=270^{\circ}}
\end{array}\right.
$$

Due to the periodic distribution of $\varepsilon_{x}\left(B_{j}\right), \varepsilon_{x B}$ and $\varepsilon_{x}\left(B_{j}\right)$ are represented respectively as,

$$
\left\{\begin{array}{l}
\varepsilon_{x B}=\left(\left.Q_{j}\right|_{B_{j}=270^{\circ}}-\left.Q_{j}\right|_{B_{j}=90^{\circ}}\right) / 2 \\
\varepsilon_{x}\left(B_{j}\right)=Q_{j}-\varepsilon_{B z} \cos B_{j}+\varepsilon_{x B} \sin B_{j}
\end{array}\right.
$$

\subsection{Measurement Pattern In X Direction}

As shown in Figure 3 and 6(b), by utilizing (1), A' is described to $O-X Y Z$ as,

$$
\begin{aligned}
\left\{A^{\prime}\right\}_{o} & =\left\{p_{j}^{l}\right\}\left\{p_{j}^{e}\right\}\left\{s_{j}^{l}\right\}\left\{s_{j}^{e}\right\}\{A\}_{o}, \\
& =\left[\begin{array}{cccc}
1 & -\varepsilon_{x B} & 0 & 0 \\
\varepsilon_{x B} & 1 & -\varepsilon_{B z} & 0 \\
0 & \varepsilon_{B z} & 1 & 0 \\
0 & 0 & 0 & 1
\end{array}\right]\left[\begin{array}{cccc}
\cos B_{j} & 0 & \sin B_{j} & d_{x}+L \sin B_{j} \\
0 & 1 & 0 & 0 \\
-\sin B_{j} & 0 & \cos B_{j} & L \cos B_{j} \\
0 & 0 & 0 & 1
\end{array}\right] \\
& {\left[\begin{array}{cccc}
1 & -\varepsilon_{z}\left(B_{j}\right) & \varepsilon_{y}\left(B_{j}\right) & \delta_{x}\left(B_{j}\right) \\
\varepsilon_{z}\left(B_{j}\right) & 1 & -\varepsilon_{x}\left(B_{j}\right) & \delta_{y}\left(B_{j}\right) \\
-\varepsilon_{y}\left(B_{j}\right) & \varepsilon_{x}\left(B_{j}\right) & 1 & \delta_{z}\left(B_{j}\right) \\
0 & 0 & 0 & 1
\end{array}\right]\left[\begin{array}{c}
0 \\
0 \\
-L \\
1
\end{array}\right] }
\end{aligned}
$$

Where $d_{x}$ indicates the original length of the ball bar in the measurement pattern of $\mathrm{X}$ direction.

By simplifying Eq. (10) and ignoring the higher order term, the distance between $\mathrm{A}^{\prime}$ and $\mathrm{O}$ in coordinate system $O-X Y Z$ is represented as,

$L_{A O}=\sqrt{-2 L \cos B_{j} d_{x} \varepsilon_{y}\left(B_{j}\right)+2 \cos B_{j} d_{x} \delta_{x}\left(B_{j}\right)+d_{x}^{2}+2 \sin B_{j} d_{x} \delta_{z}\left(B_{j}\right)}$

Since $L_{A^{\prime} O}$ is equal to the actual length of the ball bar in the $j$-th position, Eq. (12) can be obtained.

$$
\sqrt{-2 L \cos B_{j} d_{x} \varepsilon_{y}\left(B_{j}\right)+2 \cos B_{j} d_{x} \delta_{x}\left(B_{j}\right)+d_{x}^{2}+2 \sin B_{j} d_{x} \delta_{z}\left(B_{j}\right)}
$$$$
=d_{x}+\Delta d_{x}
$$ 
Where $\Delta d_{x}$ indicates the length variation of the ball bar from the initial position to the $j$-th position in the measurement pattern of $\mathrm{X}$ direction.

Then, Equation (13) is obtained by simplifying (12) and ignoring the higher order term.

$-L \cos B_{j} \varepsilon_{y}\left(B_{j}\right)+\cos B_{j} \delta_{x}\left(B_{j}\right)+\sin B_{j} \delta_{z}\left(B_{j}\right)=\Delta d_{x}$

Assuming that $P_{j}=\cos B_{j} \delta_{x}\left(B_{j}\right)+\sin B_{j} \delta_{z}\left(B_{j}\right)$, then Equation (14) is represented as follows,

$$
-L \cos B_{j} \varepsilon_{y}\left(B_{j}\right)+P_{j}=\Delta d_{x}
$$

With two different values of $L$, the following equation is obtained.

$$
\left\{\begin{array}{l}
-L_{1} \cos B_{j} \varepsilon_{y}\left(B_{j}\right)+P_{j}=\Delta d_{x 1} \\
-L_{2} \cos B_{j} \varepsilon_{y}\left(B_{j}\right)+P_{j}=\Delta d_{x 2}
\end{array}\right.
$$

According to Eq. (15), $\delta_{y}\left(B_{j}\right)$ and $Q_{j}$ are represented as respectively,

$$
\varepsilon_{y}\left(B_{j}\right)=\frac{\Delta d_{x 2}-\Delta d_{x 1}}{\left(L_{2}-L_{1}\right) \cos B_{j}}, \quad P_{j}=\frac{L_{2} \Delta d_{x 1}-L_{1} \Delta d_{x 2}}{L_{2}-L_{1}}
$$

\subsection{Measurement Pattern in Z Direction}

As shown in Figure 4 and 6(c), by utilizing (1), C' is described to $O-X Y Z$ as,

$$
\begin{aligned}
\left\{C^{\prime}\right\}_{o} & =\left\{p_{j}^{l}\right\}\left\{p_{j}^{e}\right\}\left\{s_{j}^{l}\right\}\left\{s_{j}^{e}\right\}\{C\}_{o}^{\prime} \\
& =\left[\begin{array}{cccc}
1 & -\varepsilon_{x B} & 0 & 0 \\
\varepsilon_{x B} & 1 & -\varepsilon_{B z} & 0 \\
0 & \varepsilon_{B z} & 1 & 0 \\
0 & 0 & 0 & 1
\end{array}\right]\left[\begin{array}{cccc}
\cos B_{j} & 0 & \sin B_{j} & L \sin B_{j} \\
0 & 1 & 0 & 0 \\
-\sin B_{j} & 0 & \cos B_{j} & d_{z}+L \cos B_{j} \\
0 & 0 & 0 & 1
\end{array}\right] \\
& {\left[\begin{array}{cccc}
1 & -\varepsilon_{z}\left(B_{j}\right) & \varepsilon_{y}\left(B_{j}\right) & \delta_{x}\left(B_{j}\right) \\
\varepsilon_{z}\left(B_{j}\right) & 1 & -\varepsilon_{x}\left(B_{j}\right) & \delta_{y}\left(B_{j}\right) \\
-\varepsilon_{y}\left(B_{j}\right) & \varepsilon_{x}\left(B_{j}\right) & 1 & \delta_{z}\left(B_{j}\right) \\
0 & 0 & 0 & 1
\end{array}\right]\left[\begin{array}{c}
0 \\
0 \\
-L \\
1
\end{array}\right] }
\end{aligned}
$$

Where $d_{z}$ indicates the original length of the ball bar in the measurement pattern of $\mathrm{Z}$ direction. By simplifying Eq. (17) and ignoring the higher order term, the distance between $\mathrm{C}^{\prime}$ and $\mathrm{O}$ in $O-X Y Z$ is represented as,

$L_{C O}=\sqrt{2 L \sin B_{j} d_{z} \varepsilon_{y}\left(B_{j}\right)+2 \cos B_{j} d_{z} \delta_{z}\left(B_{j}\right)-2 \delta_{x}\left(B_{j}\right) d_{z} \sin B_{j}+d_{z}^{2}}$

Since $L_{C o}$ is equal to the actual length of the ball bar in the j-th position, Eq. (19) can be obtained.

$$
\sqrt{2 L \sin B_{j} d_{z} \varepsilon_{y}\left(B_{j}\right)+2 \cos B_{j} d_{z} \delta_{z}\left(B_{j}\right)-2 \delta_{x}\left(B_{j}\right) d_{z} \sin B_{j}+d_{z}^{2}}
$$

Where $\Delta d_{z}$ indicates the length variation of the ball bar from the initial position to the $j$-th position in the measurement pattern of $\mathrm{Z}$ direction.

Then, Equation (20) is obtained by simplifying (19) and ignoring the higher order term.

$$
L \sin B_{j} \varepsilon_{y}\left(B_{j}\right)+\cos B_{j} \delta_{z}\left(B_{j}\right)-\sin B_{j} \delta_{x}\left(B_{j}\right)=\Delta d_{z}
$$

If assuming, $R_{j}=\cos B_{j} \delta_{z}\left(B_{j}\right)-\sin B_{j} \delta_{x}\left(B_{j}\right)$, Then, there is $R_{j}=\Delta d_{z}-L \sin B_{j} \varepsilon_{y}\left(B_{j}\right)$. Hence, $\delta_{z}\left(B_{j}\right)$ and
$\delta_{x}\left(B_{j}\right)$ are represented respectively as,

$$
\left\{\begin{array}{l}
\delta_{z}\left(B_{j}\right)=\cos B_{j} R_{j}+\sin B_{j} P_{j} \\
\delta_{x}\left(B_{j}\right)=\cos B_{j} P_{j}-\sin B_{j} R_{j}
\end{array}\right.
$$

So far, according to the above measured results in three different patterns in the $\mathrm{X}, \mathrm{Y}$, and $\mathrm{Z}$ direction, the five position-dependent geometric error parameters $\left(\delta_{x}(B)\right.$, $\left.\delta_{y}(B), \delta_{z}(B), \varepsilon_{x}(B), \varepsilon_{y}(B)\right)$, and two positionindependent geometric error parameters $\left(\varepsilon_{x B}, \varepsilon_{B z}\right)$ for the tilting head of five-axis machine tools can be solved totally.

\section{Conclusions}

Based on the motion condition of the tilting head of five-axis machine tools, three measurement patterns in $\mathrm{Y}$ direction, $\mathrm{X}$ direction and $\mathrm{Z}$ direction are proposed respectively using DBB. Then, the relative displacement equations between two balls of DBB in measurement patterns in $\mathrm{Y}$ direction, $\mathrm{X}$ direction and $\mathrm{Z}$ direction are established respectively on the basis of HTM and MBS theory. Finally, the geometric error parameters of the tilting head (B-axis) are identified totally. The presented method in this paper is universal and provides a reference for the error identification for the tilting head of five-axis machine tools.

\section{Acknowledgments}

This work is financially supported by the National Natural Science Foundation of China (No. 51275014), Science and Technology Major Projects of High-end CNC Machine Tools and Basic Manufacturing Equipment of China (No. 2016ZX04003001).

\section{References}

1. S H H Zargarbashi, and J.R.R Mayer 2006 Assessment of Machine Tool Trunnion Axis Motion Error using Magnetic Double Ball Bar Int J Mach Tools Manuf 46 1823-1834

2. Kwang-Il Lee, and Seung-Han Yang 2013 Measurement and verification of positionindependent geometric errors of a five-axis machine tool using a double ball-bar Int J Mach Tools Manuf 70 45-52

3. Shaowei Zhu, Guofu Ding, Shengfeng Qin, Jiang Lei, Li Zhuang, and Kaiyin Yan 2012 Integrated geometric error modeling, identification and compensation of CNC machine tools Int J Mach Tools Manuf 52 24-29

4. Yi Zhang, Jianguo Yang, and Kun Zhang 2013 Geometric error measurement and compensation for the rotary table of five-axis machine tool with double ball bar Int J Adv Manuf Technol 65 275-281

5. Ali Lasemi, Deyi Xue, and Peihua Gu 2016 Accurate identification and compensation of geometric errors 
of 5-axis CNC machine tools using double ball bar. Meas Sci. Technol. 27055004

6. Jianxiong Chen, Shuwen Lin, Xiaolong Zhou, and Tianqi Gu 2016 A ballbar test for measurement and identification the comprehensive error of tilt table Int J Mach Tools Manuf 103 1-12

7. J W Fan, J L Guan, W C Wang, Q. Luo, X L Zhang, and L Y Wang 2002 A universal modeling method for enhancement the volumetric accuracy of CNC machine tools. Journal of Materials Processing Technology 129 624-628
8. Jixiang Yang, J R R. Mayer, and Yusuf Altintas 2015 A position independent geometric errors identification and correction method for five-axis serial machines based on screw theory Int J Mach Tools Manuf 95 52-66

9. Changjun $\mathrm{Wu}$, Jinwei Fan, Qiaohua Wang and Dongju Chen 2018 Machining accuracy improvement of non-orthogonal five-axis machine tools by a newiterative compensation methodology based on the relative motionconstraint equation Int $J$ Mach Tools Manuf 124 80-98 